\title{
Relação entre alinhamento postural e desempenho motor em crianças com paralisia cerebral
}

\author{
Relationship between postural alignment and motor performance in \\ children with cerebral palsy
}

\author{
Andréa Baraldi Cunha ${ }^{1}$, Graziela Jorge Polido ${ }^{2}$, Geruza Perlato Bella ${ }^{3}$, Daniela Garbellini ${ }^{4}$, Carlos Alberto Fornasari ${ }^{5}$
}

Estudo desenvolvido na Clínica de Fisioterapia da Unimep Universidade Metodista de Piracicaba, Piracicaba, SP, Brasil

1 Fisioterapeuta especialista em Reabilitação Infantil; mestranda em Fisioterapia na

Universidade Federal de São

Carlos, São Carlos, SP

2 Fisioterapeuta especialista em Reabilitação Infantil

3 Profa. Ms. do Curso de Especialização em Reabilitação Infantil da Faculdade de Ciências Médicas da Universidade Estadual de Campinas, Campinas, SP

4 Profa. Ms. do Curso de Fisioterapia da Unimep

5 Prof. Dr. do Curso de Fisioterapia da Unimep

ENDEREÇO PARA CORRESPONDÊNCIA

Andréa Baraldi Cunha R. Marechal Deodoro 1140 ap. 131 Centro

15400-000 Olímpia SP e-mail:

andreabaraldi@gmail.com

Trabalho apresentado no XVII Congresso Brasileiro de Fisioterapia, 2007, com publicação de resumo.

APRESENTAÇÃO jul. 2008

ACEITO PARA PUBLICAÇÃO fev. 2009
Resumo: O objetivo deste estudo foi verificar se há correlação entre alinhamento postural e desempenho motor em crianças com paralisia cerebral (PC), além de compará-las com crianças de desenvolvimento motor típico. Participaram 14 crianças com PC tipo espástico, entre 4 e 12 anos, classificadas nos níveis III, IV e $\checkmark$ no sistema de classificação de função motora ampla (GMFCS); e 20 com idades entre 4 e 8 anos e desenvolvimento motor adequado, que constituíram o grupo controle. Foi avaliado o alinhamento de lordose cervical e cifose torácica na postura sentada por meio de fotometria; o desempenho motor foi avaliado pelo índice de função motora ampla (Gross motor function measure, GMFM) apenas nas dimensões sentar (B) e ficar em pé (D). O subgrupo de PC nível III obteve maiores escores no GMFM do que o dos níveis IV e V, com diferença significativa nas dimensões $B$ $(p=0,00)$ e $\mathrm{D}(p=0,016)$. Quanto ao alinhamento postural, os dois subgrupos de PC apresentaram menor angulação da lordose cervical do que o GC, com diferença significativa; também foram medidos ângulos menores da cifose torácica nos subgrupos PC, sendo que o subgrupo dos níveis IV e $V$ apresentou diferença significativa tanto em relação ao outro subgrupo PC quanto ao controle. Foi encontrada correlação positiva $(r=0,748)$ entre o desempenho motor e o alinhamento postural nos subgrupos de PC, mostrando que, quanto melhor o alinhamento postural, melhor o desempenho motor dessas crianças.

Descritores: Avaliação; Criança; Curvaturas da coluna vertebral; Paralisia cerebral

ABSTRACT: The purpose was to search for a correlation between postural alignment and motor performance of children with cerebral palsy (CP), and to compare them to typical development children. Twenty of these, aged 4 to 8 years old, formed the control group (CG); and 14 children with $\mathrm{CP}$, aged 4 to 12 years old, were divided into two subgroups (level III, and levels IV and V) according to their classification by the Gross Motor Function Classification System. Cervical lordosis and thoracic kyphosis angles in sitting posture were assessed by photometry; motor performance was assessed at dimensions B (sitting) and D (standing) of the Gross motor function measure (GMFM). PC level III subgroup had higher GMFM scores than levels IV and $V$ subgroup, with significant differences at the $B(p=0.00)$ and $D(p=0.016)$ dimensions. As to posture alignment, significantly lesser cervical lordosis angles were found at both PC subgroups when compared to control group; both subgroups also showed lesser thoracic kyphosis angles; significant differences were found when comparing CP levels IV and V subgroup both to level III subgroup and to CG. Also found was a positive correlation between motor performance and posture alignment in children with $C P(r=0.748)$, showing that the better the postural alignment, the better the motor performance in these children.

KEY words: Cerebral palsy; Child; Evaluation; Spinal curvatures 


\section{INTRODUÇÃO}

Paralisia cerebral (PC) pode ser definida, de acordo com um consenso internacional, como uma desordem de movimento e postura devido a um defeito ou lesão do encéfalo em desenvolvimento $^{1,2}$. O problema motor dos indivíduos com PC origina-se de disfunção do sistema nervoso central, que interfere diretamente no desenvolvimento do controle postural contragravidade e impede o desenvolvimento motor normal ${ }^{3}$. A disfunção no controle postural interfere nas atividades funcionais diárias, sendo um dos problemas principais nessa população ${ }^{4,5}$.

Considerando o modelo de Classificação Internacional de Funcionalidade, Incapacidade e Saúde proposta pela Organização Mundial de Saúde, a PC geralmente interfere no funcionamento do sistema musculoesquelético no que se refere à função de órgãos e sistemas ${ }^{6}$. Nesse nível, os distúrbios da motricidade e do tônus se manifestam por características específicas, como a falta de controle sobre os movimentos, modificações adaptativas do comprimento muscular e, em alguns casos, podem levar a deformidades ósseas. Isso ocorre nos períodos em que a criança apresenta ritmo acelerado de desenvolvimento, comprometendo o processo de aquisição de marcos motores básicos (rolar, sentar, engatinhar, andar) e, também, nas atividades de vida diária (tomar banho, alimentar-se, vestir-se) $)^{7,8}$.

Deformidades musculoesqueléticas associadas ao desalinhamento de tronco prejudicam o desenvolvimento da criança, acarretam alterações na marcha e até mesmo a funcionalidade na postura sentada ${ }^{9,10}$. Há também uma associação entre a direção da escoliose e obliqüidade pélvica na PC, sendo que a convexidade leva a alterações nos movimentos do tronco, sugerindo que a alteração postural está relacionada à dificuldade de ativação da musculatura de forma adequada ${ }^{11}$.

Limitações no desempenho de atividades e tarefas do cotidiano das crianças com PC estão relacionadas a deficiências neuromotoras que comprometem seu controle postural. Assim, a dificuldade em manter a postura em pé relaciona-se à pequena superfície de contato, que aumenta a demanda do sistema de controle postural. Consequentemente, muitas crianças com PC desempenham mais atividades diárias na postura sentada, que oferece uma situação de maior estabilidade e menor grau de liberdade para ser controlado ${ }^{5}$. Crianças com PC se beneficiam de terapias no contexto do controle postural, melhorando assim a qualidade do movimento, desde que não haja alterações posturais fixas ${ }^{12}$.

Considerando que a PC é uma desordem de movimento e postura e que alterações posturais podem interferir no desempenho motor, requerem-se estudos que relacionem esses fatores, para que a intervenção em crianças com PC esteja baseada em evidência, desenvolvendo um plano de tratamento adequado que permita não só prevenção de deformidades como também atividades funcionais com melhor qualidade.

O objetivo deste estudo foi verificar se há correlação entre o alinhamento postural e o desempenho motor de crianças com PC do tipo espástico, bem como comparar o desempenho motor dessas crianças com as de desenvolvimento motor adequado.

\section{METODOLOGIA}

Este estudo foi aprovado pelo Comitê de Ética da Universidade Estadual de Campinas.

Foram selecionadas 34 crianças de forma não-aleatória, com base em critérios de inclusão previamente determinados para cada grupo. Quatorze crianças com PC do tipo espástica foram divididas em dois subgrupos, de acordo com o sistema de classificação de função motora ampla (Gross motor function classification system, GMFCS ${ }^{13}$ ), sendo cinco diplégicas (grupo de PC nível III) e nove quadriplégicas (grupo de PC níveis IV e V), de ambos os sexos, atendidas na Clínica de Fisioterapia da Unimep. As crianças que se locomoviam com algum dispositivo foram classificadas como no nível III e as que não se locomoviam, nos níveis IV e V. Para participação no grupo de PC, os critérios de inclusão foram: crianças entre 4 e 12 anos, com diagnóstico médico compro- vado de PC, que tivessem capacidade de se manter sentadas de forma independente ou com apoio do terapeuta; e cujos responsáveis assinassem o termo de consentimento livre e esclarecido (TCLE). Os critérios de não-inclusão foram ter dificuldade de entendimento de ordens simples e hipotonia. O Quadro 1 resume as características desse grupo. A média de idade foi de 7,7 $\pm 3,1$ anos.

O GMFCS é um sistema de classificação utilizado para crianças com PC baseado no movimento auto-iniciado, com ênfase no sentar e andar, contendo cinco níveis diferenciados pela limitação funcional e necessidade de assistência externa $^{13}$. As crianças classificadas no nível III do GMFCS necessitam de um dispositivo auxiliar para marcha e as dos níveis IV e V têm limitações funcionais graves, principalmente na postura em pé.

Quadro 1 Características das crianças com PC

\begin{tabular}{|ccccc|}
\hline Paciente Idade* & Sexo & Topografia & Nível \\
\hline 1 & 4 & F & Quadriplegia & V \\
2 & 4 & M & Quadriplegia & V \\
3 & 4 & M & Quadriplegia & V \\
4 & 5 & F & Quadriplegia & V \\
5 & 6 & F & Diplegia & III \\
6 & 6 & M & Quadriplegia & IV \\
7 & 7 & M & Diplegia & III \\
8 & 8 & M & Diplegia & III \\
9 & 8 & M & Diplegia & III \\
10 & 9 & M & Quadriplegia & V \\
11 & 12 & M & Diplegia & III \\
12 & 12 & F & Quadriplegia & IV \\
13 & 12 & F & Quadriplegia & IVI \\
14 & 12 & F & Quadriplegia & V \\
\hline
\end{tabular}

* em anos; $\dagger$ = segundo o GMFCS, sistema de classificação de função motora ampla; $F=$ feminino; $M=$ masculino

Assim, as crianças com PC foram classificadas de acordo com sua limitação funcional na postura em pé e na marcha por meio do GMFCS, sendo subdivididas em dois subgrupos, um com crianças do nível III ( $n=5)$ e outro com as crianças dos níveis IV e V ( $\mathrm{n}=9)$.

Constituindo o grupo controle (GC), foram recrutadas em escolas da cidade de Piracicaba 20 crianças com idades

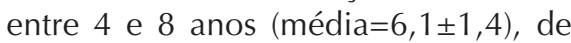
ambos os sexos, que não apresentavam 
diagnóstico de PC, com desenvolvimento motor típico, nascidas a termo com idade gestacional de 37 a 41 semanas e seis dias, sem qualquer tipo de complicação pré, peri ou pós-natal, com marcha independente, sem alterações posturais graves e cujos responsáveis assinaram o TCLE.

\section{Procedimentos}

Foi realizada uma análise de corte transversal das avaliações de desempenho motor funcional e do alinhamento na postura sentada.

Para avaliar o desempenho motor, foi utilizado o índice de função motora ampla Gross motor function measure (GMFM), que avalia o quanto de um item uma criança pode realizar (em vez de medir a qualidade de desempenho na atividade). O GMFM consiste em 88 itens agrupados em cinco diferentes dimensões ${ }^{14}$, atribuindo-se pontos a cada item. Quanto maior o escore obtido, meIhor o desempenho da criança. Foram utilizadas somente as dimensões $B$, sentar (escore máximo=60) e D, ficar em pé (escore máximo=39) nos dois subgrupos de PC, para efeito de padronização.

O alinhamento postural da coluna foi avaliado por fotometria, que é uma metodologia de interpretação dos valores obtidos a partir de uma fotografia, sendo um instrumento de caráter quantitativo que permite uma avaliação confiável dos desalinhamentos apresentados pelo segmento corporal em análi$\mathrm{se}^{15,16}$. As crianças de ambos os grupos foram fotografadas uma única vez na vista lateral do lado dominante, sentadas em um banco, com a mão dominante no ombro oposto, adução de ombro e flexão de cotovelo, flexão de $90^{\circ}$ de joelho com $10^{\circ}$ de abdução de coxofemoral, os pés afastados em relação à linha do ombro e calcanhares alinhados.

Para fotografar os sujeitos foi utilizada uma câmera fotográfica PhotoPC (750Z, Megapixel Zoom Digital Câmera, Epson). A distância entre o tripé da câmera fotográfica e o simetrógrafo foi de $180 \mathrm{~cm}$, e a altura da objetiva foi ajustada à altura da cicatriz onfálica de cada criança.

O programa Corel Draw (v.8) foi utilizado para quantificar em graus o ali-

Tabela 1 Pontuação no GMFM (média \pm desvio padrão) de função motora dos dois subgrupos de crianças com PC nas duas dimensões avaliadas

\begin{tabular}{lccc}
\hline Dimensão & PC níveis IV e V & PC nível III & \multicolumn{1}{c}{$p$} \\
\hline Sentar (B) & $28,2 \pm 5,0$ & $55,0 \pm 2,3^{*}$ & 0,00 \\
Ficar em pé (D) & $3,09 \pm 1,1$ & $12,5 \pm 3,4^{*}$ & 0,016 \\
\hline
\end{tabular}

Tabela 2 Ângulos (em ${ }^{\circ}$, média \pm desvio padrão) das curvaturas cifose torácica e lordose cervical nos dois subgrupos de crianças com PC e no grupo controle (GC)

\begin{tabular}{lcc}
\hline Grupo & Cifose torácica & Lordose cervical \\
\hline GC $(n=20)$ & $159,3 \pm 5,65$ & $137,35 \pm 7,18$ \\
PC nível III $(n=5)$ & $150,5 \pm 11,24$ & $107,75 \pm 3,77^{*}$ \\
PC níveis IV-V $(n=9)$ & $133,73 \pm 17,15^{* \dagger}$ & $112,55 \pm 12,09^{*}$ \\
\hline
\end{tabular}

nhamento postural. Na vista lateral, os pontos de referência utilizados foram os ápices de cada curvatura encontrados por meio de linhas guias traçadas verticalmente e horizontalmente no software. Por meio desses pontos, os ângulos das curvaturas foram mensurados ${ }^{16}$. Foram feitas duas medições em cada foto (pelo mesmo avaliador) e calculado seu valor médio. Quando a diferença das duas medidas excedeu os critérios aceitos (três graus de diferença entre elas), uma terceira medida foi realizada e a mais discrepante, descartada ${ }^{17}$. Foi avaliado o alinhamento da lordose cervical e da cifose torácica na postura sentada. Não foi avaliada a lordose lombar, pois as crianças com PC não apresentam essa curvatura fisiológica, somente uma cifose tóraco-lombar.

\section{Análise dos dados}

Para comparação dos escores de desempenho motor entre os dois subgrupos

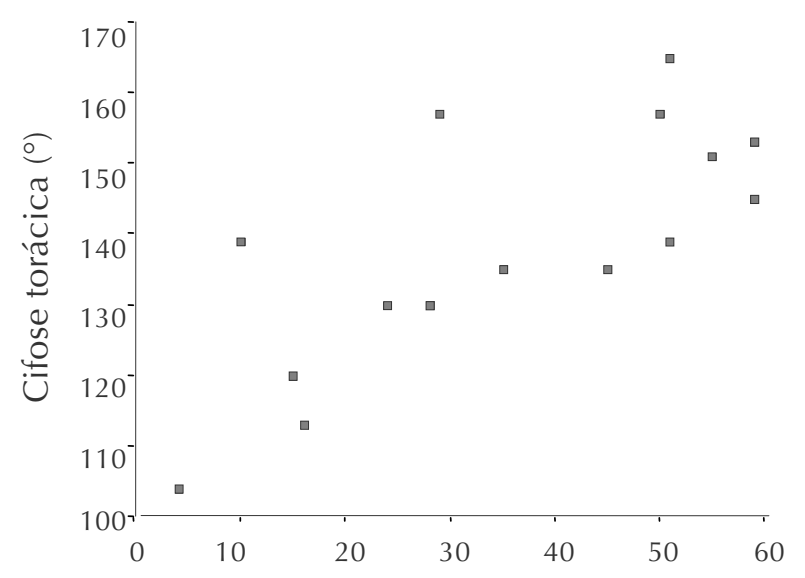

Pontuação na dimensão B (sentar) no teste de função motora

Gráfico 1 Correlação entre cifose torácica e função motora na dimensão sentar no grupo de crianças com PC de crianças com PC (nível III e níveis IV e V) foi utilizado o teste t. O nível de significância foi fixado em $p=0,05$. Para comparar os ângulos do alinhamento postural entre os dois subgrupos de crianças com PC e o grupo controle, foi utilizada a análise de variância Anova $(p<0,05)$ seguida de teste de Tukey. As análises foram realizadas utilizando o programa estatístico R versão 2.7. Para verificar a correlação entre a angulação da cifose torácica e o desempenho motor (apenas na dimensão B, sentar) nos dois subgrupos de crianças com PC foi aplicado o teste de correlação de Pearson (o coeficiente $r$ varia de $-1,0$ a 1,0 e, quanto mais próximo de 1,0, mais forte é a correlação).

\section{RESULTADOS}

A Tabela 1 mostra a pontuação média de função motora dos dois subgrupos de crianças com PC, evidenciando mai- 
ores escores do subgrupo nível III, com diferença significativa entre ambos nas dimensões sentar e ficar em pé.

Quanto aos ângulos das curvaturas, as crianças dos dois subgrupos de PC apresentaram menor angulação da lordose cervical, com diferença significativa $(p<0,05)$ quando comparadas ao GC, porém não foi encontrada diferença significativa entre os dois subgrupos. Quanto à angulação da cifose torácica, também foi encontrada angulação menor, com diferença significativa $(p<0,05)$ do subgrupo de PC níveis IV e $\mathrm{V}$ tanto em relação ao GC quanto ao subgrupo PC nível III (Tabela 2).

Quanto à correlação entre alinhamento postural e função motora na dimensão sentar nos dois subgrupos de crianças com PC, constatou-se correlação significativa $(r=0,748 ; p=0,01)$, sugerindo que, quanto melhor o alinhamento postural, melhor a função motora na dimensão avaliada (Gráfico 1).

\section{DISCUSSÃO}

Nas crianças com PC, foi encontrada uma correlação positiva entre o ângulo de cifose torácica e o desempenho motor na postura sentada, mostrando que, quanto melhor o alinhamento postural, melhor o desempenho motor dessas crianças nessa posição.

No que se refere ao alinhamento postural, foi encontrada diferença significativa nos ângulos de lordose cervical entre os dois subgrupos de PC comparados ao GC, que apresenta desenvolvimento motor e alinhamento postural adequados. A menor angulação nas crianças com PC pode ser propiciada pelo desequilíbrio muscular.

Nota-se também diferença significativa na cifose torácica no grupo de PC níveis IV e $\mathrm{V}$ quando comparado tanto ao grupo de PC nível III como ao GC. Os resultados mostram que crianças do grupo PC nível III apresentaram angulação de cifose torácica semelhante ao GC, o que é coerente com o melhor controle postural que essas crianças apresentam. O pior alinhamento das crianças do grupo PC níveis IV e V está provavelmente relacionado à diminuição de ativação muscular em extensores de tronco, mau posicionamento da pelve e falta de aquisição das reações de equilíbrio.

Kapandji ${ }^{18}$ afirma que, na primeira semana de vida, a criança levanta a cabeça, graças à musculatura antigravitacional do pescoço, formando a lordose cervical. Aos nove meses, quando a criança já se senta e começa a engatinhar, ocorre ativação da musculatura da região antigravitacional lombar, que molda a curvatura lombar. Na PC, a falta de controle postural resulta em ativação anormal da musculatura, causando a manutenção da postura cifótica e ineficientes estratégias de movimento ${ }^{19}$. Além da ativação anormal da musculatura, a fraqueza muscular é um dos fatores agravantes das alterações posturais dessas crianças $^{20}$.

Como mencionado, não foi possível comparar a lordose lombar entre os grupos de PC e GC. Não é possível mensurar essa angulação em crianças com PC porque estas apresentam cifose tóraco-lombar que pode ser justificada pela retroversão pélvica. Sullivan et al. ${ }^{21}$ mostram que a prevalência da retroversão pélvica em PCs é de $37 \%$, o que leva ao aumento da cifose e ao comprometimento da função motora na postura sentada. A retroversão pélvica na PC está associada à retração de flexores e rotadores internos de quadris e é um dos fatores que leva a criança a sentar-se sobre o sacro, levando o corpo para frente e flexionando a coluna, o que provoca postura cifótica ${ }^{10,21,22}$. Outros autores afirmam que, aos 10 anos de idade, a criança já apresenta as curvaturas fisiológicas semelhantes às dos adultos ${ }^{18}$. $\mathrm{E}$ que na posição em pé a coluna lombar está em posição de lordose; na posição sentada, a pelve e o sacro estão rodados posteriormente e a curvatura lombar está diminuída ${ }^{18}$. Isso explica que, além da retroversão pélvica, a postura sentada pode ter influenciado a ausência de lordose lombar das crianças com PC.

Estudos têm evidenciado que as deformidades e falta de ativação muscular geram limitações e uma menor capacidade de realizar tarefas. Segundo Howle $^{3}$, crianças que não apresentam funcionalidade nos membros superiores apresentam flexão da coluna, o que, com o tempo, provocará uma cifose es- trutural com hipertonia flexora de pescoço e tórax, ficando os quadris em flexão. Crianças com PC frequentemente apresentam amplitude restrita de movimento em muitas articulações, incluindo tornozelo, joelho e quadril. As contraturas dos músculos dessas articulações resultam em posturas atípicas quando o sujeito está sentado e em pé. As posturas habituais influenciam a forma pela qual os músculos são recrutados e coordenados para a recuperação da estabilidade $^{23}$.

Segundo Koman et al. ${ }^{9}$, as deformidades de coluna estão associadas à PC. A freqüência de escoliose na PC é de $25 \%$, sendo que 60 a $75 \%$ dos envolvidos são os que apresentam sérios comprometimentos e quadriplegia. A progressão da escoliose acarreta dor, interfere na função motora e comprometimento cardiopulmonar. Ostenjo et al. ${ }^{24}$ mostram que os quadriplégicos apresentam maior comprometimento referente à espasticidade que os diplégicos e, ainda, que crianças com maior comprometimento no controle motor seletivo apresentam pior desempenho na função motora ampla. Assim, é preciso prevenir que essas contraturas e/ou deformidades se instalem, corrigindo as alterações posturais que interferem diretamente na qualidade de movimento dessas crianças.

Quanto ao desempenho motor, como esperado, o subgrupo de PC nível III obteve escore mais alto na dimensão $B$ (sentar) do que o subgrupo de PC níveis IV e V, coerente com as respectivas classificações no GMFCS. Ostensjo et al. ${ }^{24}$ relatam que a maioria das crianças diplégicas se enquadra no nível III e a maioria dos quadriplégicos se enquadra no nível $V$ do GMFCS. Mancini et al.6 mostraram que crianças com nível mais grave de PC apresentam desempenho inferior às de comprometimento leve em todas as áreas funcionais. Também como esperado, o subgrupo de PC nível III apresenta melhor desempenho motor na postura de pé, já que nas crianças dos níveis IV e $\mathrm{V}$ a deficiência motora limita a funcionalidade na postura em pé. Ostensjo et al..$^{25}$ constataram que crianças com comprometimento moderado (nível III) apresentam mais atividades referentes à mobilidade que aquelas com limitações graves. Voorman et al. ${ }^{26} \mathrm{e}$ 
Bjornson et al. ${ }^{27}$ mostram ainda que crianças com muita espasticidade, como as quadriplégicas, com pior controle seletivo, fraqueza muscular, limitações no quadril e extensão de joelho, mostram curso de comprometimento da função motora menos favorável que crianças menos afetadas, principalmente na mobilidade.
O presente estudo tem limitações devido ao número reduzido de sujeitos.

\section{CONCLUSÃO}

Considerando que o alinhamento postural é a base para um adequado controle de movimento, este estudo mostrou que crianças com paralisia cerebral classificadas no GMFCS como nível III apresentam melhor desempenho motor e alinhamento postural que aquelas nos níveis IV eV; e, ainda, foi encontrada correlação positiva entre essas duas variáveis, mostrando que, quanto melhor o alinhamento postural, melhor o desempenho motor dessas crianças.

\section{REFERENCIAS}

1 Bax M, Goldstein M, Rosenbaum P, Leviton A, Paneth $\mathrm{N}$, Dan B, et al. Proposed definition and classification of cerebral palsy. Dev Med Child Neurol. 2005;44:571-6.

2 Rosenbaum P, Paneth N, Leviton A, Goldstein M, Bax $M$, Damiano D, et al. A report: the definition and classification of cerebral palsy. Dev Med Child Neurol. 2007;109(Suppl):8-14.

3 Howle JM. Neuro-developmental treatment approach: theoretical foundations and principles of clinical practice. Laguna Beach, CA: NDTA; 2000.

4 Graaf-Peters VB, Blauw-Hospers CH, Dirks T, Bakker H, Blos AF, Hadders-Agra M. Development of postural control in typically developing children and children with cerebral palsy: possibilities for intervention? Neurosci Biobehav Rev. 2007;31:1191-200.

5 Brogren $\mathrm{E}$, Algra $\mathrm{MH}$, Forssberg $\mathrm{H}$. Postural control in sitting children with cerebral palsy. Neurosci Biobehav Rev. 1998;22(4):591-6.

6 Mancini MC, Fiúza PM, Rebelo JM, Magalhães LC, Coelho ZAC, Paixão ML, et al. Comparação do desempenho de atividades funcionais em crianças com desenvolvimento normal e crianças com paralisa cerebral. Arq NeuroPsiquiatr. 2002;60(2):446-52.

7 Allegretti ALC, Mancini MC, Schwartzman JS. Estudo do desempenho funcional de crianças com paralisia cerebral diparética espástica utilizando o Pediatric Evaluation of Disability Inventory (PEDI). Arq Bras Paralis Cerebral. 2004;1(1):35-40.

8 Mancini MC, Alves ACM, Schaper C, Figueiredo EM, Sampaio RF, Coelho SAC, et al. Gravidade da PC e desempenho funcional. Rev Bras Fisioter. 2004; 8(3):253-60.

9 Koman LA, Smith BP, Shilt JS. Cerebral palsy. Lancet. 2004;363(15):1619-31.

10 Holmes KJ, Michael SM, Thorpe SL, Solomonidis SE. Management of scoliosis with special seating for the non-ambulant spastic cerebral palsy population: a biomechanical study. Clin Biomech. 2003;18:480-7.
11 Porter D, Michael S, Kirkwood C. Patterns of postural deformity in non-ambulant people with cerebral palsy: what is the relationship between the direction of scoliosis, direction of pelvic obliquity, direction of windswept hip deformity and side of hip dislocation? Clin Rehabil. 2007;21(12):1087-96.

12 Donker FS, Ledebt A, Roerdink M, Savelsbergh GJP, Beek PJ. Children with cerebral palsy exhibit greater and more regular postural sway than typically developing children. Exp Brain Res. 2008;184(3):363-70.

13 Palisano R, Rosenbaum P, Walter S, Russel D, Wood E, Galuppi B. Gross motor function classification system for cerebral palsy. Dev Med Child Neurol. 1997;39(4):214-23.

14 Russell D, Rosenbaum P, Growland C, Hardy S, Lane M, Plews N, et al. Gross motor function measure manual. 2nd ed. Toronto: McMaster University; 1993. Chap.: Administration and scoring, p.2-19.

15 Vieira ER. Análise da confiabilidade de equipamentos e métodos para medir o movimento de flexão anterior da coluna lombar [dissertação]. São Carlos: Depto. de Fisioterapia, Universidade Federal de São Carlos; 2002.

16 Abrão SD, Fornasari CA. Avaliação da posição da cabeça e plano de Frankfurt na disfunção da ATM por meio de fotometria. Rev Bras Odontol. 2005;62:82-4.

17 Bolstad G, Benum B, Rokne A. Antropometry of Norwegian light industry and office workers. Appl Ergon. 2001;32(3):239-46.

18 Kapandji IA. The physiology of the joints: the trunk and vertebral column. Edinburgh: Churchill Livingstone; 2000.

19 Casady RL, Nichols-Larsen DS. The effect of hippotherapy on ten children with cerebral palsy. Pediatr Phys Ther. 2005;17(1):72-5.

20 Ohata K, Tsuboyama T, Haruta T, Ichihashi N, Kato T, Nakamura T.Relation between muscle thickness, spasticity, and activity limitations in children and adolescents with cerebral palsy. Dev Med Child Neurol. 2008;50:152-6. 


\section{Referências (cont.)}

21 Sullivan RO, Walsh M, Jenkinson A, Brien TO. Factors associated with pelvic retraction during gait in cerebral palsy. Gait Posture. 2007;25:425-31.

22 Jones MW, Morgan E, Shelton JE. Primary care of the child with cerebral palsy: a review of systems (Part II). J Pediatr Health Care. 2007;21:226-37.

23 Shumway-Cook A, Woollacott MH. Controle motor: teoria e aplicações práticas. 2a ed. São Paulo: Manole; 2003.

24 Ostensjo S, Calberg EB, Vollestad NK. Motor impairments in young children with cerebral palsy: relationship to gross motor function and everyday activities. Dev Med Child Neurol. 2004;46:580-9.
25 Ostensjo S, Calberg EB, Vollestad NK. Everyday functioning in young children with cerebral palsy: functional skills, caregiver assistance and modifications of the environment. Dev Med Child Neurol. 2003;45:603-12.

26 Voorman JM, Dallmeijer AJ, Knol DL, Lankhorst GJ, Becher JG. Prospective longitudinal study of gross motor function in children with cerebral palsy. Arch Phys Med Rehabil. 2007;88(7):871-6.

27 Bjornson KF, Belza B, Kartin D, Logsdon R, McLaughlin JF. Ambulatory physical activity performance in youth with cerebral palsy and youth who are developing typically. Phys Ther. 2007:87(3):248-60. 